\title{
Sex and Gender Differences in Acute Pediatric Diarrhea: A Secondary Analysis of the DHAKA Study
}

\author{
Angela F. Jarman ${ }^{1,2 *}$, Sara E. Long ${ }^{2}$, Sarah E. Robertson², Sabiha Nasrin ${ }^{3}$, Nur Haque Alam ${ }^{3}$, \\ Alyson J. McGregor ${ }^{1}$, Adam C. Levine ${ }^{1,2}$ \\ ${ }^{1}$ Department of Emergency Medicine, Warren Alpert School of Medicine, Brown University, Providence, RI, USA \\ ${ }^{2}$ Center for Evidence Synthesis in Health, School of Public Health, Brown University, Providence, RI, USA \\ ${ }^{3}$ Infectious Diseases Division, International Centre for Diarrhoeal Disease Research (icddr,b), Dhaka, Bangladesh
}

\section{ARTICLE INFO}

Article History

Received 15 February 2018

Accepted 28 April 2018

\section{Keywords}

Bangladesh

diarrheal illness

gender

pediatric

\begin{abstract}
Pediatric diarrheal disease is a significant source of morbidity and mortality in the developing world. While several studies have demonstrated an increased incidence of diarrheal illness in boys compared with girls in low- and middle-income countries (LMIC), the reasons for this difference are unclear. This secondary analysis of the dehydration: assessing kids accurately (DHAKA) derivation and validation studies included children aged $<5$ years old with acute diarrhea in Dhaka, Bangladesh. The dehydration status was established by percentage weight change with rehydration. Multivariable regression was used to compare percent dehydration, while controlling for differences in age and nutritional status. In this cohort, a total of 1396 children were analyzed; 785 were male (56.2\%) and 611 were female (43.8\%). Girls presenting with diarrhea were older than boys (median age 17 months vs. 15 months, $p=0.02$ ) and had significantly more malnutrition than boys, even when controlled for age (mean mid-upper arm circumference $134.2 \mathrm{~mm}$ vs. $136.4 \mathrm{~mm}, p<0.01)$. The mean percent dehydration did not differ between boys and girls after controlling for age and nutrition status $(p=0.25)$. Although girls did have higher rates of malnutrition than boys, measures of diarrhea severity were similar between the two groups, arguing against a cultural bias in care-seeking behavior that favors boys.
\end{abstract}

(C) 2018 Atlantis Press International B.V.

This is an open access article under the CC BY-NC license (http://creativecommons.org/licenses/by-nc/4.0/).

\section{INTRODUCTION}

Despite the decreasing global burden of pediatric diarrheal disease over the past few decades, it remains a leading cause of death in children under the age of 5 years $[1,2]$. Worldwide, there are an estimated 957 million cases of diarrheal illness and 499,000 deaths in children under 5 years of age each year [2]. Acute diarrhea is the fourth leading cause of death and accounts for $9 \%$ of all deaths in this population [2], with the vast majority of deaths occurring in children under 2 years of age [3]. In Bangladesh, despite a $60 \%$ decrease in the child mortality rate from 2005 to 2015 , over 3800 deaths in children under 5 years of age were attributed to acute diarrheal disease in 2015 [2]. The estimated economic burden of pediatric diarrheal illness is significant as well, particularly in low- and middle-income countries (LMIC), which are disproportionately affected [1-4]. Diarrheal illness is thus a significant contributor to disability-adjusted life years, mainly due to years of life lost. In 2015 more than 45 billion disability-adjusted life years were attributed to diarrheal disease in children under 5 years of age [2].

Although the common causes of acute diarrheal disease in children are thought to be well understood, there is emerging epidemiologic evidence of sex and gender differences, with boys being disproportionally affected compared with girls. A recent systematic review found that among studies with sex data available, boys

*Corresponding author. Email: angela.jarman@gmail.com were overrepresented compared with girls with acute diarrheal disease; data were similar for acute pulmonary infections [3]. The majority of the studies that found sex and gender differences were conducted in LMICs like Bangladesh [5-9]. A recent study from Ethiopia even found boys to have 2.52 times [95\% confidence interval (CI) 1.28-4.93] the adjusted odds of having acute diarrhea as compared with girls [9]. Other studies, including a recent systematic review, however, found gender-stratified global prevalence rates for pediatric diarrhea to be similar [10-12].

While the cause of the increase in diarrheal disease in boys is unclear, several hypotheses have emerged that attribute the variance to gender-based factors, such as cultural and environmental factors, or sex-based biological factors. The cultural theory postulates that boys are treated differently than girls due to cultural beliefs that value boys more highly, therefore making them more likely to receive timely diagnosis and treatment [13-15]. Environmental theories suggest different exposures by gender, for example, older boys may be allowed more freedom to roam from home, or go to work with fathers, unequally exposing them to infectious pathogens $[6,7,9]$. The biological hypothesis assumes that there may exist pathophysiologic sex differences between girls and boys with regard to acute diarrhea that make boys more susceptible [6].

Despite numerous studies demonstrating an increased incidence of diarrheal illness in boys compared with girls in LMICs, the reason for this difference remains unclear. The current study aims 
to understand if boys and girls with acute diarrheal illness differ in severity on presentation, based on a planned secondary analysis of a large data set from Bangladesh. The purpose of the original study was to develop and validate the dehydration: assessing kids accurately (DHAKA) score, a clinical decision tool to assess and guide the treatment of dehydrated children aged 5 years or younger in low resource settings $[16,17]$. We hypothesize that within this data set, boys are overrepresented due to gendered cultural norms in this region regarding health-seeking behavior that favor medical care for male children, and that boys, therefore, will have less severe dehydration than girls upon presentation.

\section{MATERIALS AND METHODS}

\subsection{Study Design}

This study was a planned secondary analysis of pooled data from the DHAKA derivation and validations studies. These studies were originally designed to derive and validate a clinical dehydration tool for use in low-resource settings. Each was a prospective cohort study; detailed study protocols have been previously published [16,17]. Ethical approval was obtained from the International Centre for Diarrhoeal Disease Research (icddr,b), Bangladesh Ethical Review Committee, and the Rhode Island Hospital (Lifespan) Institutional Review Board. For all eligible children, research staff explained the risks and benefits of the study and obtained informed consent from the parent or guardian of the child in the local language, Bengali.

\subsection{Study Setting and Population}

The study cohorts included children under the age of 60 months presenting to the short-stay rehydration unit at the icddr,b in Dhaka, Bangladesh between February 2014 and June 2014 (derivation study) and March 2015 and May 2015 (validation study). The short stay unit at our study hospital functions as a rehydration unit specifically for patients with acute diarrhea. There, patients are generally admitted for hours to a few days, and either discharged home, or transferred to the long-stay unit if their illness persists or they have other complications. The icddr,b is a large research hospital that provides free services to Dhaka and the surrounding areas, which includes a catchment area of 17 million [18]. Children were eligible for inclusion in the studies if they presented with acute diarrhea, defined by the World Health Organization (WHO) as three or more loose stools per day for $<14$ days $[19,20]$.

\subsection{Data Collection}

After screening, eligible children were randomized for inclusion in the study 24 hours a day, by choosing blue (selected) or white (not selected) marbles from a blind pouch. If enrolled, these children were assessed for multiple clinical signs of dehydration and weighed on arrival by one or more general practice nurses. In addition to demographic data, a mid-upper arm circumference (MUAC) was obtained in all enrolled children. MUAC has been shown to outperform $z$-scores as a measure of malnutrition in dehydrated children of both sexes; therefore, this was used as a proxy for nutrition status [21]. Enrolled children were resuscitated per standard icddr,b protocol and were reweighed every 8 hours during the course of their rehydration, until a stable weight was achieved. Stable weight was achieved when the two highest consecutive weights differed by $<2 \%$. The dehydration status was established by percentage weight change with rehydration, calculated as follows: Percent dehydration $=[$ (stable weight - admission weight)/stable weight $] \times 100$. The dehydration status was collected as both a continuous variable (percent dehydration) and categorized according to standard guidelines (severe dehydration $>9 \%$, some dehydration $=3-9 \%$, and no dehydration $<3 \%$ ).

\subsection{Data Analysis}

For the secondary analysis, we merged data from both DHAKA studies. We performed complete-case analysis, excluding children missing data for any variables included in the final model (gender, age, MUAC, percent dehydration). The largest proportion of data, approximately $8 \%$, was missing for dehydration status due to loss to follow up from patients discharged prior to achieving a stable weight who did not return for a follow-up weight check. In the derivation study, participants that lost $>3 \%$ of their body weight during admission were excluded from analysis. Those patients are included in this secondary analysis; however, we did perform a sensitivity analysis to demonstrate that their inclusion did not have a significant effect on the results. Figure 1 details the complete analytic sample. The complete data set was analyzed to evaluate for any sex-based differences with a primary outcome of percent dehydration. Other outcome variables were chosen a priori based on prior studies. Hypothesis testing was conducted for each outcome variable by gender. For categorical variables, chi-square tests and Wilcox Rank Sum tests were used, and a Student $t$-test was used for continuous variables.

We used multivariate linear regression to compare percent dehydration for boys and girls. Given significant baseline differences in nutritional status (MUAC) and age between girls and boys, these were controlled in the final model. This adjusted regression model included children with complete data for gender, dehydration status, age, and nutrition. We used additional linear regression models to compare the secondary outcomes of total days of diarrhea prior to presentation and number of episodes of diarrhea in the preceding day for boys and girls; the term episode, in each of the DHAKA studies, refers to a discrete loose bowel movement. This model was also adjusted for age and nutrition. A logistic regression model was also analyzed according to the children's degree of dehydration, a categorical outcome variable.

In addition, data were also analyzed by age strata, given the hypothesis that gender may be associated with different environmental exposures $[5,6]$. We hypothesized that these exposures may vary by age, and particularly by ambulatory status and gender, and therefore created a model that categorized children by age group into preambulatory ( $<12$ months), toddler (13-36 months), and older children (36-60 months). A logistic regression was used to analyze these age-stratified groups by gender, again controlling for nutrition status.

\section{RESULTS}

A total of 1396 children were analyzed in the pooled cohort, which included 850 from the derivation study and 546 from the 


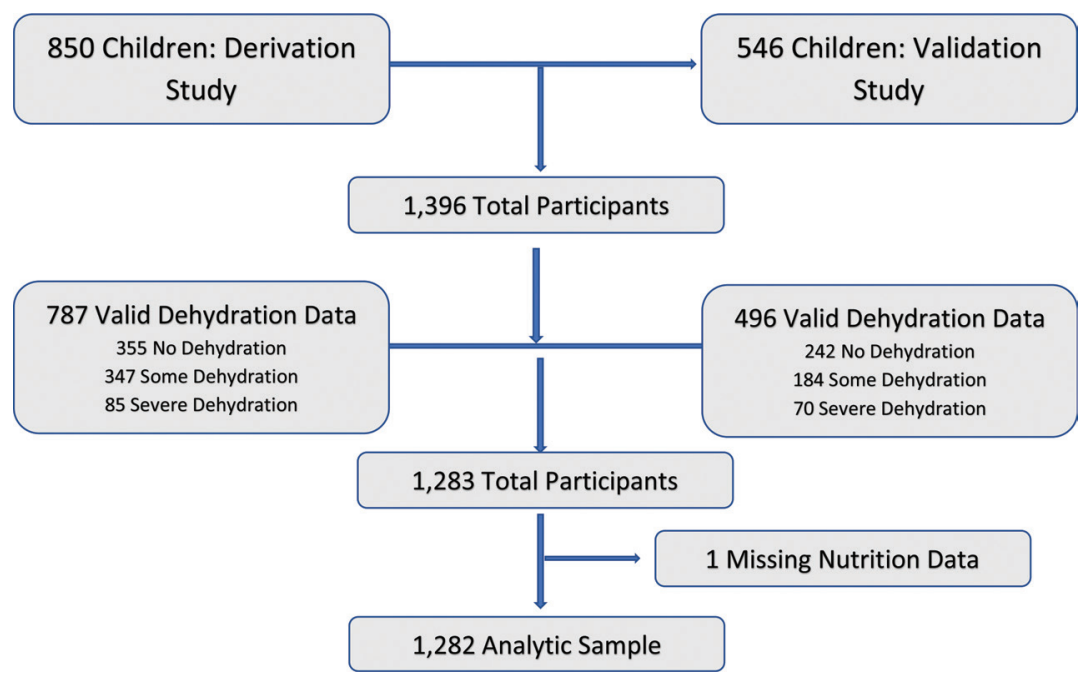

Figure 1 Flowchart detailing participant selection

Table 1 Baseline characteristics of study participants by cohort (derivation, validation, and cumulative)

\begin{tabular}{|c|c|c|c|}
\hline & $\begin{array}{c}\text { Derivation } \\
\text { cohort } \\
(n=850)\end{array}$ & $\begin{array}{c}\text { Validation } \\
\text { cohort } \\
(n=546)\end{array}$ & $\begin{array}{l}\text { Cumulative } \\
(n=1396)\end{array}$ \\
\hline $\begin{array}{l}\text { Age (months), } \\
\text { median (IQR) }\end{array}$ & $15(9,30)$ & $16(9,30)$ & $16(9,30)$ \\
\hline \multicolumn{4}{|l|}{ Sex, $n(\%)$} \\
\hline Female & $368(43.3)$ & $243(44.5)$ & $611(43.8)$ \\
\hline Male & $482(56.7)$ & $303(55.5)$ & $785(56.2)$ \\
\hline MUAC (cm), mean (SD) & $134.6(13.1)$ & $136.7(13.1)$ & $135.4(13.1)$ \\
\hline \multicolumn{4}{|l|}{ Nutritional status, $n(\%)$} \\
\hline No malnutrition & $618(72.8)$ & $431(78.9)$ & $1049(75.2)$ \\
\hline Moderate & $174(20.5)$ & $86(15.8)$ & $260(18.6)$ \\
\hline Severe & $57(6.7)$ & $29(5.3)$ & $86(6.2)$ \\
\hline $\begin{array}{c}\text { Days of diarrhea, } \\
\text { median (IQR) }\end{array}$ & $2(1,4)$ & $2(1,3)$ & $2(1,3)$ \\
\hline $\begin{array}{l}\text { Episodes in previous } \\
24 \mathrm{~h} \text {, median (IQR) }\end{array}$ & $15(10,20)$ & $10(8,18)$ & $15(10,20)$ \\
\hline \multicolumn{4}{|l|}{ Diarrhea type, $n(\%)$} \\
\hline Watery & $496(58.5)$ & $400(73.3)$ & $896(64.3)$ \\
\hline Rice water & $348(41.0)$ & $143(26.2)$ & $491(35.2)$ \\
\hline Bloody & $4(0.5)$ & $3(0.5)$ & $7(0.5)$ \\
\hline $\begin{array}{l}\text { Percent dehydration, } \\
\text { mean (SD) }\end{array}$ & $3.9(4.3)^{\mathrm{a}}$ & $4.2(4.4)^{\mathrm{b}}$ & $4.0(4.4)^{\mathrm{c}}$ \\
\hline \multicolumn{4}{|l|}{ Dehydration category, $n(\%)$} \\
\hline None & $355(45.1)^{\mathrm{a}}$ & $242(48.8)^{\mathrm{b}}$ & $597(46.5)^{c}$ \\
\hline Some & $347(44.1)$ & $184(37.1)$ & $531(41.4)$ \\
\hline Severe & $85(10.8)$ & $70(14.1)$ & $155(12.1)$ \\
\hline
\end{tabular}

${ }^{\mathrm{a}} n=787 ;{ }^{\mathrm{b}} n=496 ;{ }^{\mathrm{c}} n=1283$; IQR, interquartile range; MUAC, mid-upper arm circumference; $\mathrm{SD}$, standard deviation.

validation study. Baseline characteristics by cohort are shown in Table 1, which includes demographic data, along with our primary and secondary outcome measures. As shown, the median age was 16 months, with an interquartile range (IQR) of 9-30. A total of $56.2 \%$ of the cohort was male and nearly a quarter $(24.8 \%)$ of these children suffered from moderate to severe malnutrition. They presented with a median of 2 days of diarrhea (IQR 1-3), and 15 episodes of diarrhea in the preceding 24 hours (IQR 10-20). Most children (64.3\%) presented with watery type diarrhea and the mean percent dehydration for all children was $4.0 \%$.
As seen in Table 2, which shows outcome measures by sex, boys were significantly overrepresented when compared with girls in all cohorts. A total of 785 boys were analyzed, representing $56.2 \%$ of study participants (95\% CI 53.6-58.9), compared with $43.8 \%$ or 611 girls (95\% CI 41.1-46.4). Girls presenting with diarrhea were significantly older than boys (median age 17 months vs. 15 months, $p=0.02$ ). Girls were also significantly more malnourished than boys, as evidenced by their smaller MUAC (mean $134.2 \mathrm{~mm}$ vs. $136.4 \mathrm{~mm}$, $p<0.01$ ). Boys and girls did not, however, have significant differences with regard to degree of dehydration. For those with complete data $(n=1282)$, the mean percent dehydration was $4.0 \%$ for boys compared with $3.9 \%$ for girls $(p=0.65)$; boys and girls also had a similar distribution across the categories of dehydration. Although boys and girls presented after the same number of days of diarrhea [median 2, IQR 1-4 (boys), IQR 1-3 (girls)], boys did have slightly more episodes of diarrhea in the preceding 24 hours than girls (median 15 vs. $13, p=0.03$ ). There were no significant differences with regard to the types of diarrhea with which boys and girls presented.

Table 3 shows regression coefficients for our primary and secondary outcomes. Both an adjusted and an unadjusted model are shown; the adjusted model includes age and MUAC. As seen in Table 3, sex was not a significant predictor of percent dehydration, even when controlled for age and nutrition status $(p=0.25)$. While there was no effect on days of diarrhea prior to presentation, the regression model did find that girls had fewer episodes of diarrhea in the prior 24 hours than boys $(p=0.02)$. Additional regression results are shown in Table 4, which show the odds ratio for having each level of dehydration for girls compared with boys. Sex was not a significant predictor in any category of dehydration, even when controlled for age and MUAC.

As noted previously, no significant difference was found among children missing data and those included in the final model of the derivation study. In the validation study, those who were lost to follow up did tend to be older, but other significant differences were not found [16]. Given the purpose of this study, we did include 16 children that were excluded in the original derivation study due to significant weight loss. We performed a sensitivity analysis in which we repeated our analyses with these children excluded and did not find any significant differences in our results. 
Table 2 Baseline and outcome metrics by sex, cumulative cohort

\begin{tabular}{lccc}
\hline Characteristics & Boys $(\boldsymbol{n}=\mathbf{7 8 5})$ & Girls $(\boldsymbol{n}=\mathbf{6 1 1})$ & $\boldsymbol{p}$-Value \\
\hline Total percent $(95 \%$ CI) & $56.2(53.6-58.9)$ & $43.8(41.1-46.4)$ & \\
Age (month), median (IQR) & $15(9-30)$ & $17(10-30)$ & 0.02 \\
MUAC (cm), mean (95\% CI) & $136.4(135.5-137.3)$ & $134.2(133.1-135.2)$ & $<0.01$ \\
Nutrition status & & & \\
No malnutrition, $n$ (\% of gender) & $621(79.1)$ & $428(70.2)$ & $<0.01$ \\
Moderate malnutrition & $127(16.2)$ & $133(21.8)$ & \\
Severe malnutrition & $37(4.7)$ & $49(8)$ & \\
Days of diarrhea, median (IQR) & $2(1-4)$ & $2(1-3)$ & 0.63 \\
Episodes in previous 24 hours, median (IQR) & $15(10-20)$ & $13(9-20)$ & 0.03 \\
Diarrhea type, $n$ (\%) & & & \\
Watery & $512(65.3)$ & $384(63.0)$ & 0.25 \\
Rice water & $270(34.4)$ & $221(36.2)$ & \\
Bloody & $2(0.3)$ & $5(0.8)$ & 0.65 \\
Percent dehydration, mean (95\% CI) ${ }^{\mathrm{a}}$ & $4.0 \%(3.7-4.4)$ & $3.9 \%(3.56-4.27)$ & \\
Dehydration category & & & \\
None, \% of gender & $334(46.1)$ & $263(47.1)$ & 0.43 \\
Moderate & $295(40.8)$ & $236(42.2)$ & \\
Severe & $95(13.1)$ & $60(10.7)$ & \\
\hline
\end{tabular}

${ }^{\mathrm{a}} n=1283 ; \mathrm{CI}$, confidence interval; IQR, interquartile range; MUAC, mid-upper arm circumference.

Table 3 Adjusted and unadjusted regression coefficient for girls compared with boys with acute diarrhea in patients $<60$ months

\begin{tabular}{lcccc}
\hline Outcome variables & Unadjusted coefficient $(\mathbf{9 5 \%} \mathbf{C I})$ & $\boldsymbol{p}$-Value & Adjusted coefficient $(\mathbf{9 5 \%} \mathbf{C I})^{\mathrm{a}}$ & $\boldsymbol{p}^{\text {-Value }}$ \\
\hline Percent dehydration & $-0.001(-0.006,0.004)$ & 0.65 & $-0.003(-0.008,0.002)$ & 0.25 \\
Days of diarrhea & $-0.012(-0.21,0.18)$ & 0.90 & $-0.009(-0.20,0.18)$ & 0.93 \\
Episodes in previous 24 hours & $-1.07(-1.94,-0.20)$ & 0.02 & $-1.06(-1.93,-0.18)$ & 0.02 \\
\hline
\end{tabular}

${ }^{\mathrm{a}} \mathrm{n}=1282$; CI, confidence interval.

Table 4 Adjusted and unadjusted odds ratio of dehydration for girls compared with boys with acute diarrhea in patients $<60$ months

\begin{tabular}{lcccc}
\hline Outcome variables & Unadjusted OR $(\mathbf{9 5 \%}$ CI $)$ & $\boldsymbol{p}$-Value & Adjusted OR (95\% CI) & $\boldsymbol{p}$-Value \\
\hline Dehydration category & & & & \\
No dehydration & $1.02(0.82,1.26)$ & 0.85 & $1.13(0.90,1.41)$ & 0.29 \\
Moderate & $1.05(0.84,1.30)$ & 0.69 & $0.99(0.79,1.24)$ & 0.93 \\
Severe & $0.79(0.56,1.11)$ & 0.18 & $0.76(0.54,1.07)$ & 0.17 \\
\hline
\end{tabular}

${ }^{\mathrm{a}} n=1282$; CI, confidence interval; OR, odds ratio.

\section{DISCUSSION}

This study sought to further understand the overrepresentation of boys compared with girls with acute diarrhea in Bangladesh, which is not explained by census or hospital data [18,22]. This finding is in line with an emerging trend in several studies from LMICs in Africa and Asia.

We hypothesized that girls would have greater degrees of dehydration and markers of illness severity than boys in our cohort, indicating a cultural care-seeking bias toward boys. In fact, however, we found dehydration indices to be similar by sex, thus indicating alternative etiologies. While our data did not suggest a care-seeking bias, many of the studies that have found a male predominance of disease were conducted in historically patriarchal countries that undervalue women relative to men, which suggests that gender may be an important social determinant of disease.

Understanding this trend is complex, as the role of social determinants of health and disease, such as gender, socio-economic status (SES) and related sanitation practices, are often not included in many studies. Public health data suggest that unsafe water and sanitation practices are the most important factors putting children at risk of acute diarrheal disease, which is infectious [23]. It is plausible that environmental exposures that differ by gender and age may account for this difference in disease incidence. This has not been well studied and may be regional, but additional data will be necessary to further understand the ways in which boys' and girls' exposures differ and how that contributes to their gender-specific morbidity and mortality.

It is possible that there is a sex-based difference in the pathophysiology of acute pediatric diarrhea that we do not yet understand. Some epidemiologic data from the USA also find that boys are disproportionally affected by diarrheal illness, and that they are more likely to be hospitalized or die [24-26]. This would suggest that there may be a sex-based physiologic mechanism at work given the relative rarity of unsafe water or sanitation in the USA. The mortality of acute diarrheal illness is significantly lower in the USA than in LMICs [27], however, and therefore sex-specific data on 
transmissibility, infectivity, and mechanisms of immunity are not well studied.

In order to more fully understand sex- and gender-based differences in the prevalence of acute pediatric diarrheal illness, additional investigation is needed. While controlled trials are not feasible, cohort and case control studies can work to include socioeconomic and demographic data to further understand the role of social determinants of disease, such as gender. In addition, methodologic interventions, such as analyzing data both in aggregate and in sex-specific strata, may yield additional knowledge. Qualitative research may also prove to be a pivotal component of the body of literature on this topic so that we can more fully understand any gender bias. Finally, we should include gender-specific mortality rates for all related research to aid in understanding not only the infection rates, but outcomes by gender.

\subsection{Limitations}

Although this was a relatively large study, we lacked sufficient power to detect small sex-based differences in dehydration indices. Likewise with the age stratified groups, although we do suspect that exposures vary across age groups. Given the complicated study protocol and limitations with obtaining complete data on all participants, we were missing data on $8 \%$ of children in the final model. Based on sensitivity analysis in the original studies, this did not result in significant differences between participants who remained and those who were lost to follow up. Finally, hypotheses explaining the overrepresentation of boys compared with girls include many social determinants of the disease, about which we did not collect data; these include the demographic variables of income, household composition, parental education, and race/ethnicity. Future research should include these variables and consider a qualitative component to better understand the different exposure profiles of boys compared with girls.

\section{CONCLUSION}

In our study cohort, significantly more boys presented with acute diarrheal illness than girls. In seeking to further understand the overrepresentation of boys, we analyzed indices of illness severity, namely degree of dehydration. Girls did have higher rates of malnutrition than boys; however, measures of diarrhea severity were similar between the two groups. These results argue against a cultural bias in care-seeking behavior that favors boys. Additional investigation into the higher incidence of diarrheal disease found among boys in some LMICs may relate to other mechanisms, such as varied environmental exposures or physiologic sex differences.

\section{CONFLICTS OF INTEREST}

Funding for this study was provided by the National Institutes of Health (NIH) Fogarty International Center (FIC) (K01TW009208). The funding sources had no involvement in the design or conduct of the study or the decision to submit for publication. None of the authors have any conflicts of interest to report. All authors had full access to all the study data and had final responsibility for the decision to submit for publication.

\section{REFERENCES}

[1] Fischer Walker CL, Perin J, Aryee MJ, Boschi-Pinto C, Black RE. Diarrhea incidence in low- and middle-income countries in 1990 and 2010: a systematic review. BMC Public Health 2012;12;220.

[2] GBD Diarrhoeal Diseases Collaborators. Estimates of global, regional, and national morbidity, mortality, and aetiologies of diarrhoeal diseases: a systematic analysis for the Global Burden of Disease Study 2015. Lancet Infect Dis 2017;17;909-48.

[3] Walker CLF, Rudan I, Liu L, Nair H, Theodoratou E, Bhutta ZA, et al. Global burden of childhood pneumonia and diarrhoea. Lancet 2013;381;1405-16.

[4] Rheingans RD, Antil L, Dreibelbis R, Podewils LJ, Bresee JS, Parashar UD. Economic costs of rotavirus gastroenteritis and cost-effectiveness of vaccination in developing countries. J Infect Dis 2009;200 Suppl 1;S16-S27.

[5] Chowdhury F, Khan IA, Patel S, Siddiq AU, Saha NC, Khan AI, et al. Diarrheal illness and healthcare seeking behavior among a population at high risk for diarrhea in Dhaka, Bangladesh. PLoS One 2015;10;e0130105.

[6] Siziya S, Muula AS, Rudatsikira E. Correlates of diarrhoea among children below the age of 5 years in Sudan. Afr Health Sci 2013;13;376-83.

[7] Melo MC, Taddei JA, Diniz-Santos DR, Vieira C, Carneiro NB, Melo RF, et al. Incidence of diarrhea in children living in urban slums in Salvador, Brazil. Braz J Infect Dis 2008;12;89-93.

[8] Haque R, Mondal D, Kirkpatrick BD, Akther S, Farr BM, Sack $\mathrm{RB}$, et al. Epidemiologic and clinical characteristics of acute diarrhea with emphasis on Entamoeba histolytica infections in preschool children in an urban slum of Dhaka, Bangladesh. Am J Trop Med Hyg 2003;69;398-405.

[9] Anteneh ZA, Andargie K, Tarekegn M. Prevalence and determinants of acute diarrhea among children younger than five years old in Jabithennan District, Northwest Ethiopia, 2014. BMC Public Health 2017;17;99.

[10] Khalil I, Colombara DV, Forouzanfar MH, Troeger C, Daoud F, Moradi-Lakeh M, et al. Burden of diarrhea in the Eastern Mediterranean Region, 1990-2013: findings from the Global Burden of Disease Study 2013. Am J Trop Med Hyg 2016;95; 1319-29.

[11] Alam N, van Ginneken JK, Timaeus I. Determinants of perceived morbidity and use of health services by children less than 15 years old in rural Bangladesh. Matern Child Health J $2009 ; 13 ; 119-29$.

[12] El Arifeen S, Baqui AH, Victora CG, Black RE, Bryce J, Hoque $\mathrm{DM}$, et al. Sex and socioeconomic differentials in child health in rural Bangladesh: findings from a baseline survey for evaluating Integrated Management of Childhood Illness. J Health Popul Nutr 2008;26;22-35.

[13] Nair H, Simoes EA, Rudan I, Gessner BD, Azziz-Baumgartner E, Zhang JSF, et al. Global and regional burden of hospital admissions for severe acute lower respiratory infections in young children in 2010: a systematic analysis. Lancet 2013;381;1380-90.

[14] Pandey A, Sengupta PG, MondalSK, Gupta DN, Manna B, Ghosh S, et al. Gender differences in healthcare-seeking during common illnesses in a rural community of West Bengal, India. J Health Popul Nutr 2002;20;306-11.

[15] Mitra AK, Rahman MM, Fuchs GJ. Risk factors and gender differentials for death among children hospitalized with diarrhoea in Bangladesh. J Health Popul Nutr 2000;18;151-56. 
[16] Levine AC, Glavis-Bloom J, Modi P, Nasrin S, Atika B, Rege S, et al. External validation of the DHAKA score and comparison with the current IMCI algorithm for the assessment of dehydration in children with diarrhoea: a prospective cohort study. Lancet Glob Health 2016;4;e744-e51.

[17] Levine AC, Glavis-Bloom J, Modi P, Nasrin S, Rege S, Chu C, et al. Empirically derived dehydration scoring and decision tree models for children with diarrhea: assessment and internal validation in a prospective cohort study in Dhaka, Bangladesh. Glob Health Sci Pract 2015;3;405-18.

[18] Bardhan PK, Mustafa M. Annual Statistics of Dhaka Hospital. Dhaka, Bangladesh: International Centre for Diarrhoeal Disease Research, Bangladesh; 2012.

[19] Department of Child and Adolescent Health and Development. IMCI handbook: Integrated management of childhood illness. Geneva: World Health Organization; 2005.

[20] Department of Child and Adolescent Health and Development. The treatment of diarrhoea: A manual for physicians and other senior health workers, 4th ed., Geneva: World Health Organization; 2005.

[21] Modi P, Nasrin S, Hawes M, Glavis-Bloom J, Alam NH, Hossain MI, et al. Midupper arm circumference outperforms weight- based measures of nutritional status in children with diarrhea. J Nutr 2015;145;1582-87.

[22] National Institute of Population Research and Training - NIPORT/ Bangladesh, Mitra and Associates, ICF International. Bangladesh Demographic and Health Survey 2014. Dhaka, Bangladesh: NIPORT, Mitra and Associates, and ICF International; 2016.

[23] Black RE, Morris SS, Bryce J. Where and why are 10 million children dying every year? Lancet 2003;361;2226-34.

[24] Malek MA, Curns AT, Holman RC, Fischer TK, Bresee JS, Glass RI, et al. Diarrhea- and rotavirus-associated hospitalizations among children less than 5 years of age: United States, 1997 and 2000. Pediatrics 2006;117;1887-92.

[25] Glass RI, Lew JF, Gangarosa RE, LeBaron CW, Ho MS. Estimates of morbidity and mortality rates for diarrheal diseases in American children. J Pediatr 1991;118;S27-S33.

[26] Flores AR, Szilagyi PG, Auinger P, Fisher SG. Estimated burden of rotavirus-associated diarrhea in ambulatory settings in the United States. Pediatrics 2010;125;e191-e98.

[27] Esposito DH, Holman RC, Haberling DL, Tate JE, Podewils LJ, Glass RI, et al. Baseline estimates of diarrhea-associated mortality among United States children before rotavirus vaccine introduction. Pediatr Infect Dis J 2011;30;942-47. 